\title{
Potential Role of Proton Pump Inhibitors Against Human DRD2 Receptor in Drug Induced Hyperprolactinemia
}

\author{
MUHAMMAD ASHFAQ ${ }^{1}$, S. MOBASHER ALI ABID ${ }^{1}$, KHALID RAUF ${ }^{1}$, \\ YASSER MSA ALKAHRAMAN ${ }^{1 *}$, MUHAMMAD ZEESHAN HAROON ${ }^{2}$, \\ FAWAD AHMAD ${ }^{3}$, SAIMA IKRAM ${ }^{3}$, JAMSHAID AHMAD ${ }^{3 *}$ \\ ${ }^{1}$ Department of Pharmacy, COMSATS University Islamabad, Abbottabad Campus, Pakistan \\ ${ }^{2}$ Department of Community Medicine, Ayub Medical College, Abbottabad, Pakistan \\ ${ }^{3}$ Centre of Biotechnology \& Microbiology, University of Peshawar, Pakistan
}

\begin{abstract}
Dopamine is a catecholamine neurotransmitter that control several important functions via different dopamine receptors(D1-D5). The Dopamine (DRD2) receptor and other D2 family receptors (D3 and D4) are predominantly involved in the inhibitory activities. One vital role of dopamine receptors is its involvement in the endocrine regulations including the hormone synthesis and their secretion. The regulation of prolactin hormone is mainly controlled through DRD2 receptors. Blocking the delivery of dopamine at these DRD2 receptors will cause an increase in serum prolactin levels. PPI's are among the widely prescribed medications used for multiple gastric hypersecretory disorders and are shown to cause increase in serum prolactin level. This study focuses on computational methods to test PPIs interaction with dopamine D2 receptor through molecular docking \& dynamics studies. The $3 D$ structure of protein and the drugs were downloaded from PDB and PubChem databases. Protein and ligands were prepared followed by molecular docking. Complexes with best docking poses were then proceeded towards MD simulations of $60 \mathrm{~ns}$. Results were then analyzed. This study confirmed that there is prospective affinity between proton pump inhibitors and dopamine D2 receptor, and dynamically stable complexes are formed after drug-receptor binding. MD simulations results confirmed the binding affinity between PPIs and Dopamine D2 receptor, concluding that the use of PPIs may be involved in drug induced hyperprolactinemia and other related effects.
\end{abstract}

Keywords: Proton Pump Inhibitors, DRD2 receptor, Docking, MD Simulation, Hyperprolactinemia

\section{Introduction}

Dopamine is a monoamine catecholamine neurotransmitter that control a variety of functions including cognition, emotion, food intake, positive reinforcement, locomotor activity and endocrine system regulation in central nervous system. Dopamine also plays several functions peripherally like modulation of cardiovascular functions, vascular tone, renal functions, gastrointestinal motility, catecholamine release and hormonal secretion. Dopamine exerts these functions through dopamine receptors which has been characterized into five distinct membrane bound receptors (D1-D5). These dopamine receptors are recognized by their ligand specificity, G-protein coupling, physiological impacts and their anatomical distribution. D1 and D5 have been gathered into D1 family and D2, D3 and D4 into D2 family. The D1 family couple to Gs and intercede excitatory neurotransmission. The D2 family coupled to Gi/Go and intervene inhibitory neurotransmission [1-3].

D1 and D5 are mainly located in CNS in nucleus accumbens, the striatum, substantia nigra and olfactory bulb. All of these are implicated in the regulation of reward system, memory, learning and motor activities. Centrally D2, D3 and D4 are primarily found in the striatum and external globus pallidus, amygdala, hippocampus, Cerebral cortex and core of nucleus accumbens. Postsynaptic receptors are also affected by these receptors which exhibit extrapyramidal activities. These receptors have important role in the signaling for the survival of dopaminergic neurons and neuro developmental processes [1,4].

\footnotetext{
*email: yasser@cuiatd.edu.pk, jamshaidbiotech@yahoo.com
} 
Peripherally dopamine receptors are located in cardiovascular, renal immune and sympathetic nervous system.

One of main function of the dopaminergic system apart from the central functions, in the periphery is endocrine regulation. Prolactin is a polypeptide hormone and is in the family with growth hormone. Prolactin secretion is primarily controlled from hypothalamus by dopamine. The dopamine is released from tuberoinfundibular neurons which are terminating in the median eminence and are transported through hypophyseal portal vessels into the anterior pituitary gland. The binding of dopamine and dopamine 2 receptor (DRD2) occurs on the surface of pituitary lactotroph cell, which reduce intracellular cAMP, thus causing decrease in prolactin secretion. Any factor that causes the disruption of delivery of dopamine to the anterior pituitary will eventually results in hyperprolactinemia. Various drugs have been demonstrated to have antagonistic interactions with DRD2 receptor and causes an increase in serum prolactin level $[5,6]$. A study conducted on DRD2 knockout mice model has revealed the involvement of DRD2 in pituitary functions and as expected DRD2 knockout mice have persistent hyperprolactinemia and pituitary hyperplasia. [7] The study conducted by [8] found that prolactin level increased in third month of life in knockout animals and a chronic hyperprolactinemic state was developed throughout life.

Proton pump inhibitors are increasingly prescribed medications that inhibit $\mathrm{H}+/ \mathrm{K}+$ ATPase (an important enzyme associated with ultimate step for the secretion of Hydrochloric acid $\mathrm{HCl}$ ) in the gastric parietal cells. So proton pump inhibitors are the medications used to treat various hyper secretory stomach disorders including chronic peptic ulcer, H pylori infection, stomach outlet obstruction, GERD, gastritis, and Zollinger Ellison syndrome [9]. Since the introduction in 1989, PPIs have now become one of the most extensively prescribed class of drugs [10]. The first member of this class of drugs is Omeprazole(6-methoxy-2-(((4-methoxy-3,5-dimethylpyridin-2-yl)methyl)sulfinyl)-1H-benzo[d]imidazole). Other members includes Esomeprazole which is $\mathrm{S}$ isomer of Omeprazole ((S)-6-methoxy-2-(((4methoxy-3,5-dimethylpyridin-2-yl)methyl)sulfinyl)-1H-benzo[d]imidazole), Pantoprazole (6-(difluoromethoxy)-2-(((3,4-di- methoxypyridin-2-yl)methyl)sulfinyl)-1H-benzo[d]imidazole), Rabeprazole (2(((4-(3-methoxy-propoxy)-3-methylpyridin-2-yl)methyl)sulfinyl)-1H-benzo[d]imidazole),Lansoprazole (2-(((3- methyl-4-(2,2,2-trifluoroethoxy)pyridin-2-yl)methyl)sulfinyl)-1H-benzo[d]imidazole) and Dexlansoprazole, which is $\mathrm{R}$ isomer of the Lansoprazole ((R)-2-(((3-methyl-4-(2,2,2-trifluoroethoxy) pyridin-2-yl)methyl)sulfinyl)-1H-benzo[d]imidazole) [11]. Several studies have variable capacity of different PPI's to cross blood brain barrier. Different PPI's including omeprazole, lansoprazole and pantoprazole has been documented to cross blood brain barrier. Thus, they can have direct influence on the brain and may cause additional effects in the brain [12-14]. There are multiple evidences that PPIs can trigger hyperprolactinemia resulting in gynecomastia, galactorrhea, hormonal disturbances and sexual abnormalities [15,16]. The clinical implication of hyperprolactinemia are amenorrhea, oligomenorrhea, decrease in libido and galactorrhea in women. Men with hyperprolactinemia often represents with loss of libido, gynecomastia, erectile dysfunction and rarely galactorrhea. Sexual problems have been reported in many studies with use of PPI's particularly omeprazole $[17,18]$. Studies have demonstrated that dopamine receptor occupancy in the pituitary is the main indicator of the hyperprolactinemia $[19,20]$. Therefore, the aim of this study was to evaluate the prediction of binding affinity of PPIs with DRD2 receptor in anterior pituitary, using in silico techniques for possible prolactin secretion.

\section{Materials and methods}

Computational approach was used for this study. Overall methodology is explained in scheme 1. 


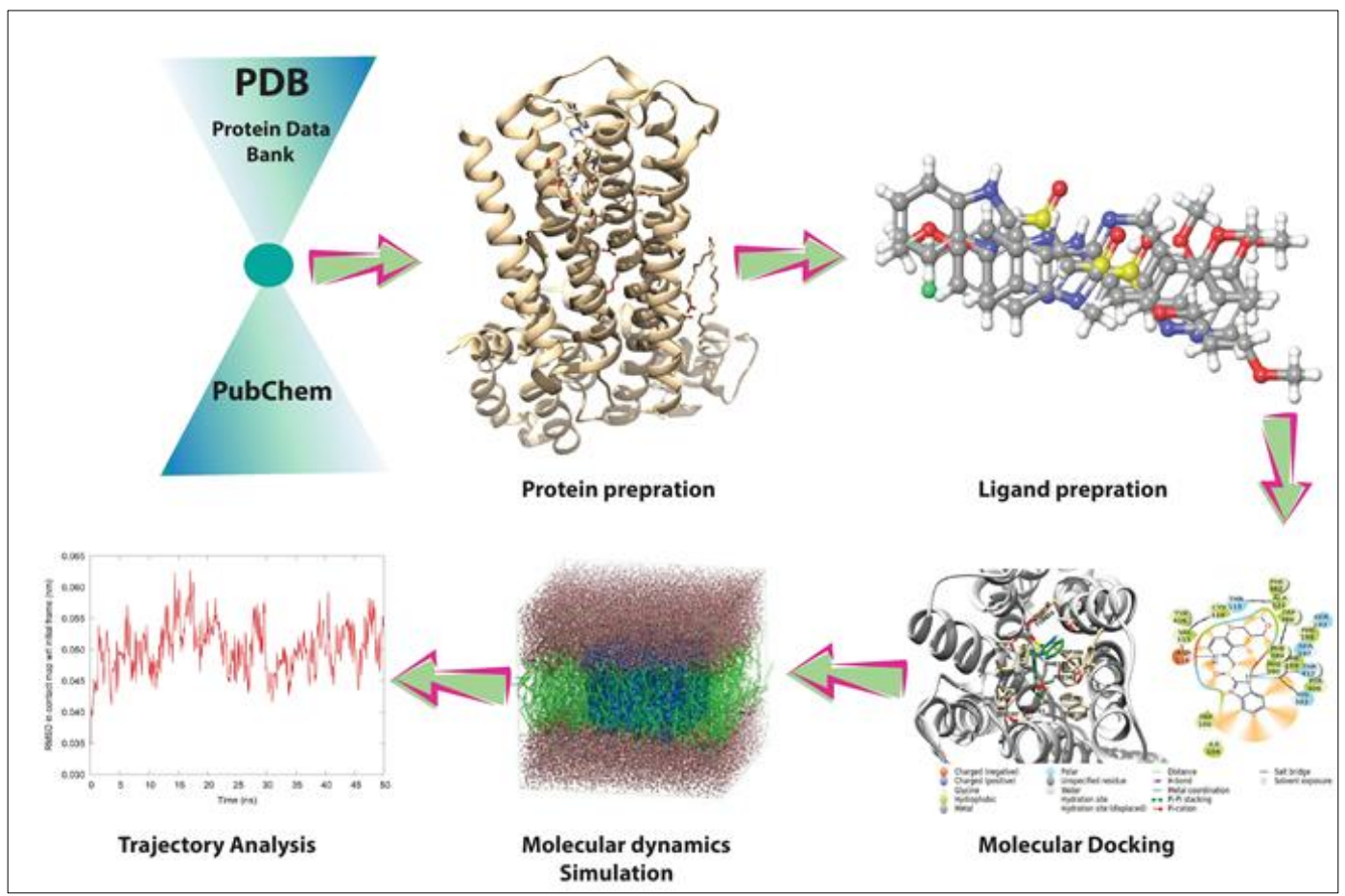

Scheme 1. Overall methodology of designed study. Protein and ligands were Downloaded from PDB and PubChem databases

\subsection{Receptor Preparation}

The DRD2 receptor 3D structure was obtained from RSCB Protein Data Bank (PDB ID 6CM4) with the resolution of $2.86 \AA$ [21]. Maestro protein preparation wizard was used for DRD2 preparation. Hydrogens were added and bond orders were allocated. The protonation states of residues were determined at the physiological $p \mathrm{H}$ of 7.4 [22,23]. The arranged structure was minimized by utilizing OPLS2005 force field. Bounded ligand was kept during protein preparation. Later on, that ligand was used for defining the binding pocket. Water molecules located around the bounded ligand within $5 \AA$ distance were kept at the binding pocket [24].

\subsection{Preparation of Ligand}

2D structures of four available PPIs were downloaded from PubChem and Lig-Prep module of maestro was used to prepare the selected ligands $[25,26]$. At the physiological $p \mathrm{H}$, protonation states of the ligands were resolute using Epik [27]. The selected PPIs are listed below in Table 1.

\subsection{Docking Procedure}

All the selected PPIs were docked in the binding pocket of DRD2 receptor to forecast their binding energies. Glide/SP docking algorithm was used for docking simulation. Grid box was made around the co-crystallized ligand to define its accurate binding pocket. Hydroxyl and thiol groups of following residues were allowed for rotation: TYR93, SER103, THR112, CYS118, THR119, SER121, SER163, SER167, TYR192, SER193, SER194, SER197, TYR199, CYS385, THR392, TYR408, SER409, THR412, TYR416 and SER419. 0.08 scaling factor was selected for VdW radii of ligand atoms with partial atomic charge. Rest of the settings were used as default. 10 poses were produced for each ligand among which the best docked conformation was selected for MD simulation analysis. The docking procedure was carried out and the required final docking energy $(\Delta G)$ was determined $[28,29]$. 
Table 1. List of the PPI drugs along with their 2D structure, molecular formula and molecular mass $(\mathrm{g} / \mathrm{mol})$

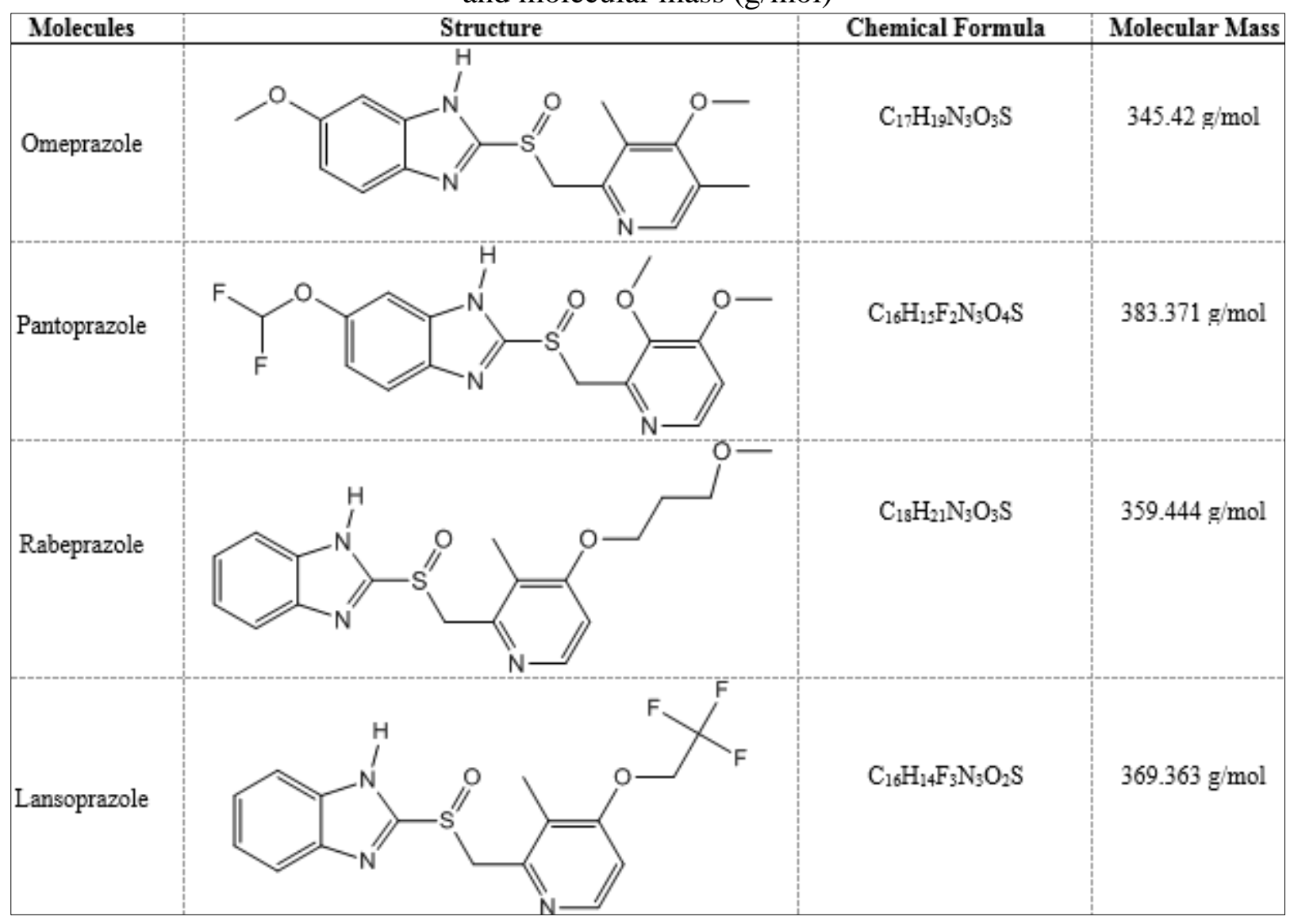

\subsection{Simulation Analysis}

Molecular Dynamics (MD) simulation was performed for a period of $60 \mathrm{~ns}$ to analyze the structural and dynamic changes in DRD2 receptor along with selected PPIs using Desmond [30]. Systems were embedded into a 1-palmitoyl-2-oleoyl-sn-glycero-3-phosphocholine (POPC) membrane bilayer, and then solvated in an orthorhombic box with layer of explicit TIP3P water molecules. OPLS2005 force field was used to calculate the systems' energies. Particle Mesh Ewald (PME) method has been used to evaluate short and long electrostatic interactions between the Van der Waals and Columbic interactions with a cut-off radius of $12.0 \AA$. Thermostat and Martyna-Tobias-Klein protocols were utilized to change the device temperature and pressure at respectively $310 \mathrm{~K}$ and $1.01325 \mathrm{~cm}[31,32]$. Time step was assigned as $2.0 \mathrm{fs}$ for all simulations. Finally, $60 \mathrm{~ns}$ MD simulation was carried out for each system.

\subsection{Binding Free Energy (MM/GBSA)}

MM/GBSA is a feasible approach to calculate the binding energy of a system. For this purpose, 100 trajectory frames were considered from the 60ns simulations. The OPLS2005 force field and VSGB 2.0 solvation model were applied [32].

\section{Results and discussions}

Molecular docking studies are very useful in evaluation of interacting mechanism between ligand and protein. Higher efficacy is reflected when there is higher binding affinity between ligand and protein receptor. The geometries of the four compounds (Omeprazole, Pantoprazole, Rabeprazole, Lansoprazole) were optimized and docking results for each PPI compound were assessed against DRD2 receptor target and only the pose having the lowest binding free energy and root mean square deviations (RMSD) were carefully chosen. Minimum docking energy $(\Delta \mathrm{G})$ in $\mathrm{Kcal} / \mathrm{mol}$ was calculated to predict 
the final Glid/SP docking score and are given in Table 2. Glid/SP docking score suggests good binding affinity between PPIs-DRD2.

In contrast to the MM/GBSA scores discussed further in this study, the docking score from Table 2 suggests that comparatively high interaction exists between Rabeprazole and DRD2 receptor followed by Pantoprazole, Lansoprazole and Omeprazole.

Table 2. List of the four selected PPI's and there corresponding docking scores in $\mathrm{Kcal} / \mathrm{mol}$

\begin{tabular}{|c|c|}
\hline Molecules & Docking score Kcal/mol \\
\hline Lansoprazole & -6.96 \\
\hline Omeprazole & -6.73 \\
Rabeprazole & -7.13 \\
Pantoprazole & -7.07 \\
\hline
\end{tabular}

One of the most important neurotransmitters, dopamine is involved in regulation of different functions in the body and if functional anomaly due to this happens, it may lead to conditions like schizophrenia, Parkinson's disease, nausea, vomiting, ADHD and depression. DRD2 is one of the five GPCRs which are regulated by dopamine, thus one of the important targets utilized by the antipsychotic drugs. Other than many ailments, the DRD2 receptor antagonism is also involved in the elevation of the prolactin level in the blood.

Risperidone binding mode has already been elaborated and as defined, the whole structure of Risperidone may be segmented into iso-oxazole, piperidine and tetrahydro-pyrido-pyrimidine moieties [19]. The deep binding pocket consisting of Cys118, Thr119, Ser197, Phe198, Phe382, Phe390 and Trp386 does show interaction with the iso-oxazole segment. The other two segments piperidine (middle) and tetrahydro-pyrido-pyrimidine interact with the hydrophobic region of the receptor pocket. ASP114 makes salt bridge with nitrogen of the piperidine ring.

Rabeprazole was analyzed for the docking pose and the interactions with the receptor amino acids. The methyl-pyridine moiety of the Rabeprazole amino site and the ASP114 showed interaction in the form of salt bridge-salt bridge formation as depicted by the Figure $(1 \& 2)$. This has been shown by the Risperidone's piperidine ring in Figure 1 and 2.

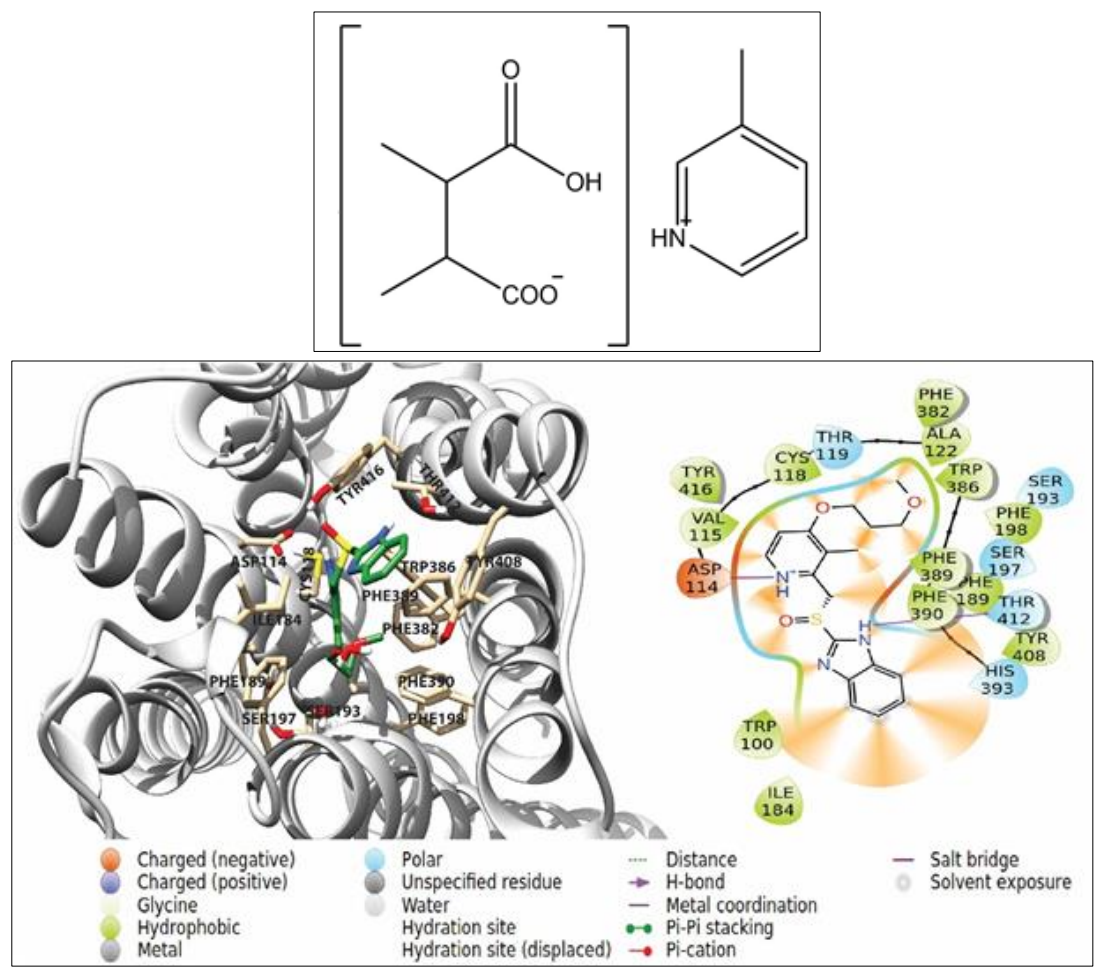

Rev. Chim., 71 (10), 2020, 182-192
Figure 1. Showing the mode of interaction of ASP114 with the methylpyridine moiety of the Rabeprazole

Figure 2. 2D and 3D interaction map of Rabeprazole in the binding pocket of dopamine D2 receptor 
Methyl-pyridine is also showing the hydrophobic interactions with different amino acids of the cavity i.e. VAL115, TYR416 and CYS118. The ethereal oxygen of the side chain attached to pyridine shows some polar interactions with THR119. The benzimidazole's hydrogen at nitrogen moiety showed Hbond interaction with the THR412.

Omeprazole when studied for the molecular interactions with the DRD2, its di-methyl and methoxy substituted pyridine moiety finds way to make salt bridge interactions with ASP114 while the hydrogen of pyridine nitrogen forms H-bond interaction with ASP114. The imidazole moiety shows hydrophobic interactions with different amino acids namely TYR416, ILE 184 ALA122 and PHE189. Pi-Pi stacking interactions are also seen with TRP386 and PHE390 Figure (3).

Lansoprazole interaction with the receptor showed almost the same conformation of the imidazole and pyridine moieties and the clear presence of ASP114 and pyridine salt bridge interaction. The triflorides showed polar interactions with THR412 and SER409. The benzimidazole shows Pi-Pi stacking interactions with TRP386 and PHE390 Figure (3).

On the other hand, Pantoprazole did not show any salt bridge interaction with ASP114. Its benzimidazole moiety showed Pi-Pi stacking with PHE386 and PHE390. Some polar interaction in the cavity were also seen with SER409, THR412, HIS393, THR119, SER197 and SER193 as shown in Figure (3).

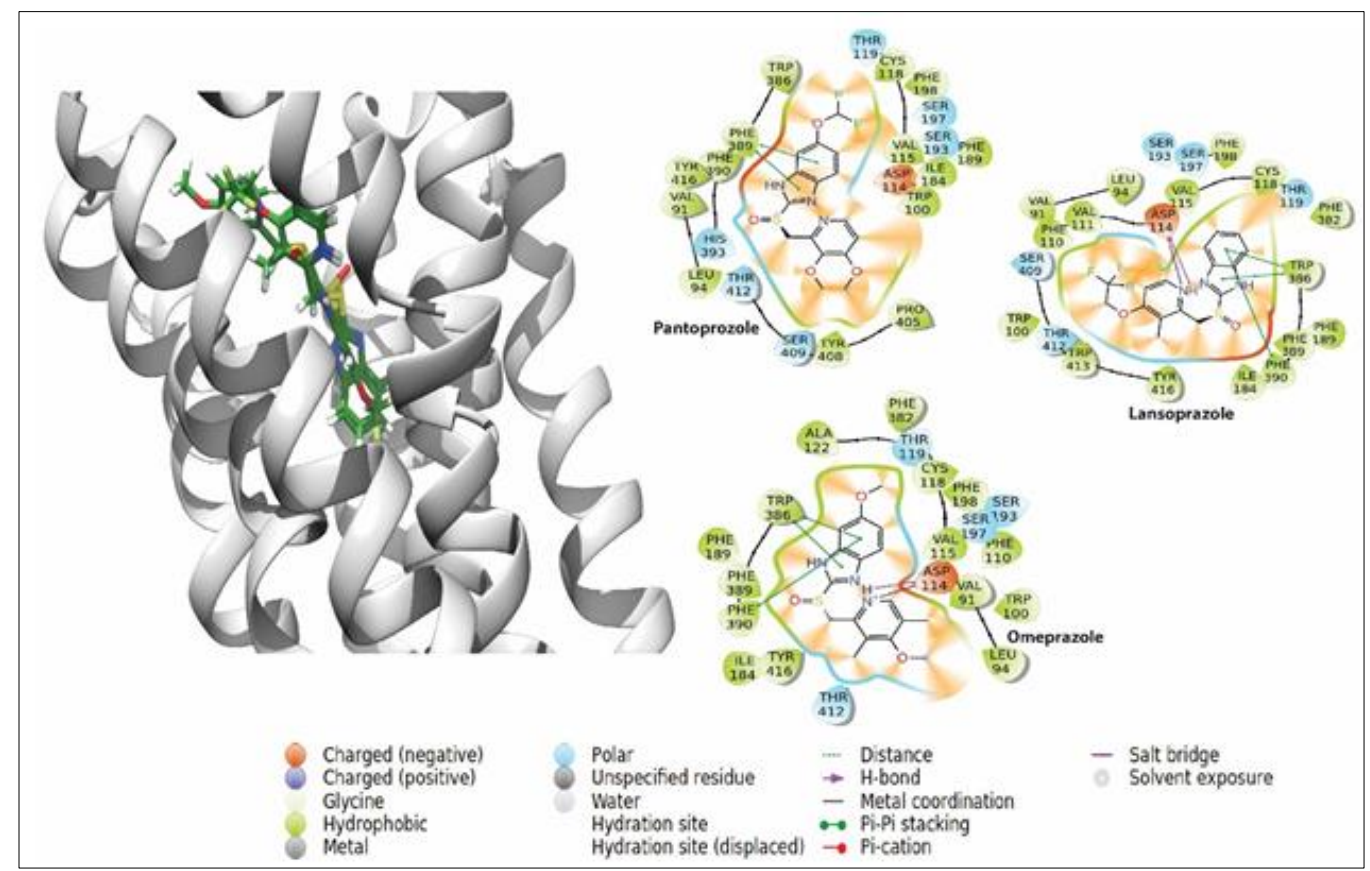

Figure 3. Pantoprazole, Lansoprazole and Omeprazole docked in the binding pocket of dopamine D2 receptor. 2D interaction maps show the interacting residues

All the three molecules, Rabeprazole, Omeprazole and Lansoprazole showed expected interactions with the DRD2 receptor. Thus, this makes these molecules to be the possible inhibitors of DRD2. This will lead to the triggering of the possible mechanism of excessive release of prolactin in the blood. This mechanism runs as the inhibition of dopaminergic receptors in the pituitary lactotroph cells which may lead to over production of the prolactin. This concludes that the patients/individuals taking these drugs as a remedy of the gastric hypersecretions may have a risk of hyperprolactinemia. So among all these four molecules, the drug Lansoprazole, seems to have the slightest interaction with the DRD2.

To examine the conformational variations of the DRD2 receptor, the root mean square deviation (RMSD) of the atomic positions with respect to the four PPI's structures was calculated. The RMSD for the backbone atoms of the DRD2 receptor-PPIs as a function of the simulation time is shown in the 
Graph (1). The plot shows pretty stable profile especially for the Lansoprazole, Pantoprazole and Rabeprazole Graph (1). These showed sufficient stability for 60 ns trajectory.

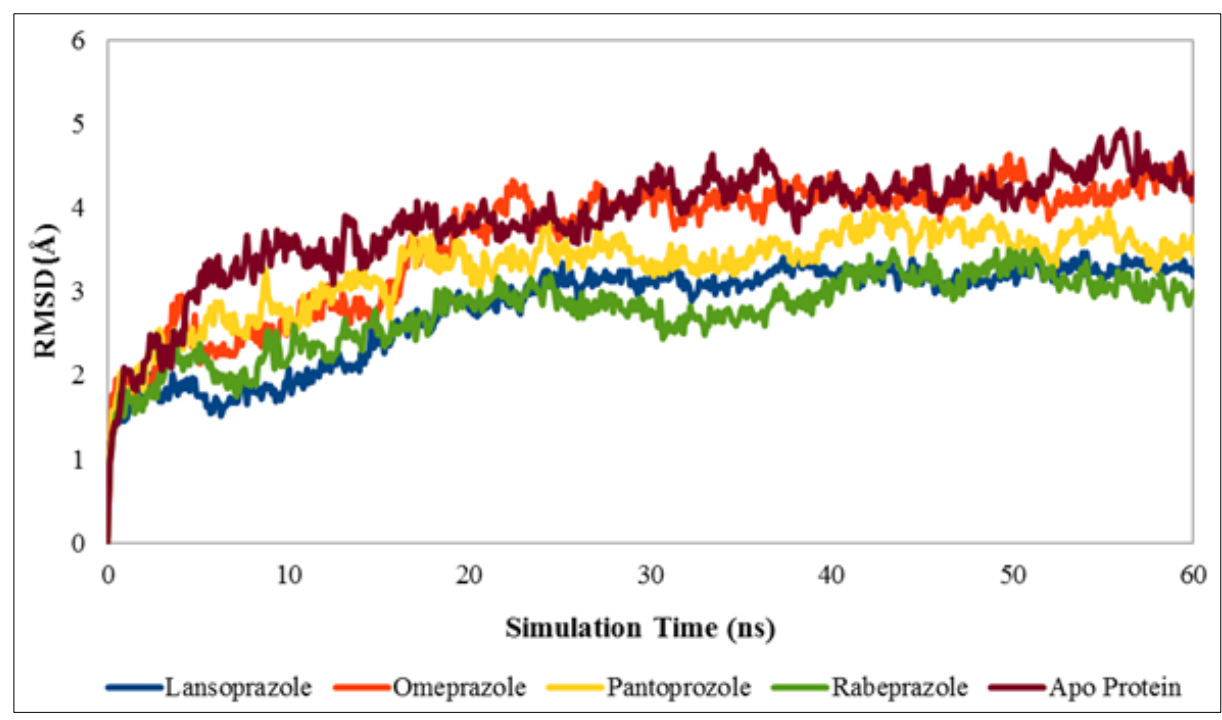

Graph 1. Graphical representation of RMSD results throughout MD simulations.

Time scale (ns) is represented at x-axis, whereas y-axis describes RMSD fluctuation in $\AA$ for protein-ligand complexes

The Lig-Fit-protein measures the translational motion of the ligand in the binding pocket of the receptor. For RMSD of Lig-Fit-protein calculations, heavy atoms of the ligand were considered. In the pro-fit mode, it is clearly indicated that Lansoprazole, Omeprazole and Rabeprazole have stable conformation in the binding pocket of receptor. At the same time, Pantoprazole showed wider fluctuations as compared to other molecules, which confirms that the Pantoprazole is less effective in its inhibitory activity against DRD2 receptor as shown in Graph (2).

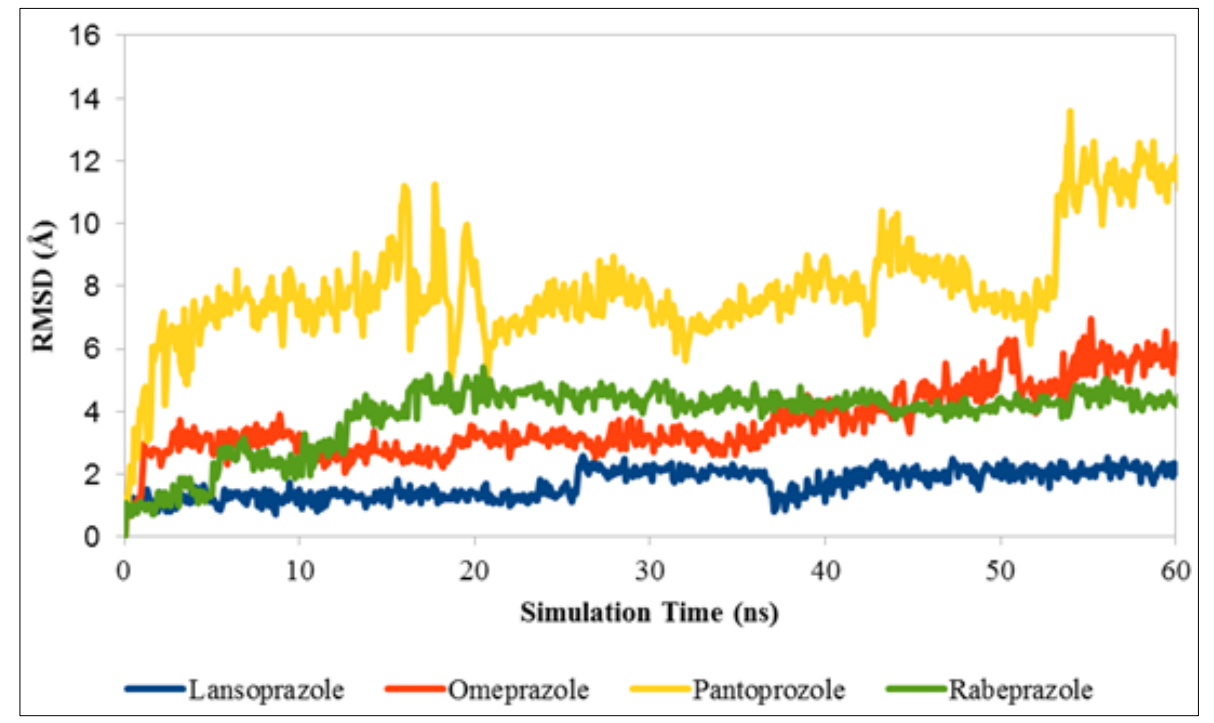

Graph 2. Lig-Fit-protein RMSD graph of the selected molecules and apo form of protein. This shows how much the ligand is deviating from the protein.

This is actually the analysis of protein ligand compatibility

To investigate the dynamic features in PPI's binding with DRD2, roote mean square fluctuations (RMSF) were also monitored along the MD trajectories. The $\mathrm{C} \alpha \mathrm{RMSF}$ values calculated as a function 
of residue number are shown in Graph (3). The RMSF graph showed that there are minor fluctuations in all of the simulation period as compared to the ligand free protein (DRD2). Rabeprazole and Omeprazole complexes showed similar fluctuating pattern as that of DRD2 (apo-protein). The highest fluctuations were observed in case of Lansoprazole.

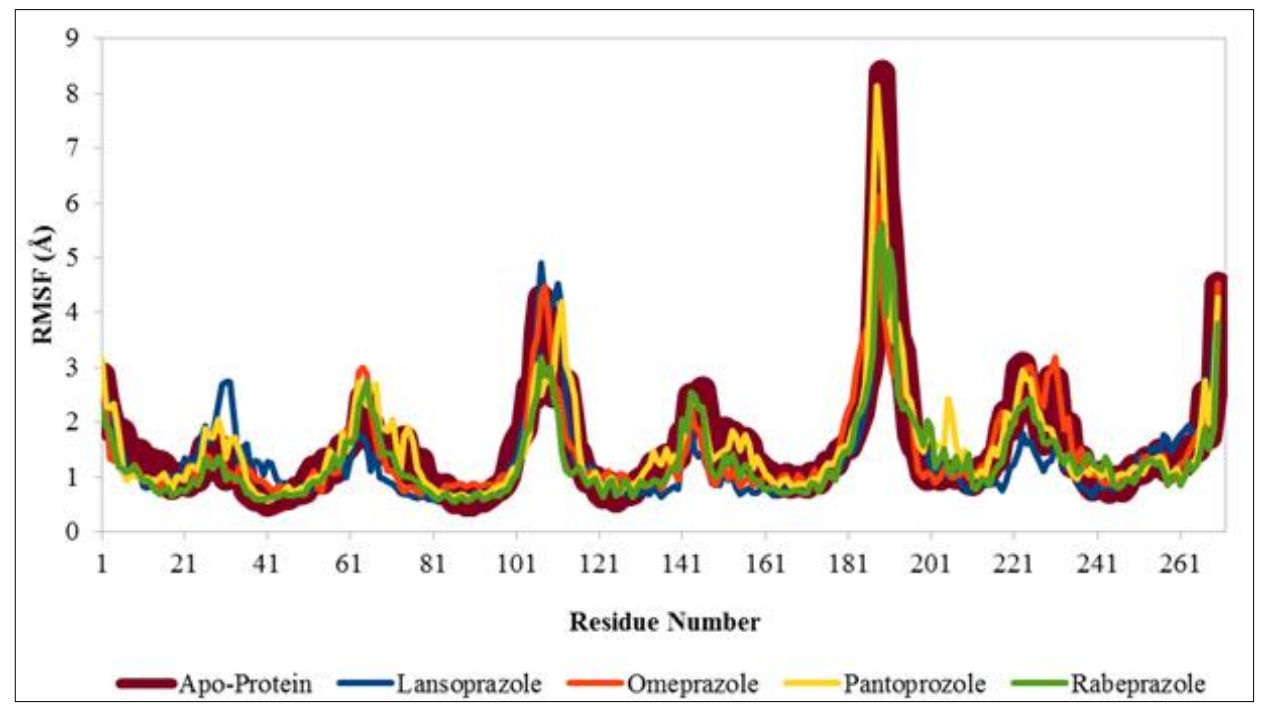

Graph 3. RMSF of protein Carbon-alpha residues throughout MD simulation time. The bold dark brown line is unbound protein's RMSF while the rest of the coloured lines represent the RMSF of drug bound protein

To investigate the relative binding affinities of all four PPIs with DRD2 receptor, MM/GBSA binding energies were also calculated for the period of $60 \mathrm{~ns}$. MM/GBSA score (in Kcal/mol), which is used to estimate the relative binding affinities for a list of ligands, was calculated for all PPI's molecules and is shown in Table 3. In addition, the ligand efficiency has also been shown to elaborate the ratio of affinities of each PPI molecule per atom to the DRD2 receptor. Table 3 suggests that high degree of interaction exists between Rabeprazole and DRD2 receptor followed by Lansoprazole, Omeprazole and Pantoprazole.

Table 3. MM/GBSA value in $\mathrm{Kcal} / \mathrm{mol}$ and ligands efficiency score of selected drugs

\begin{tabular}{|c|c|c|}
\hline Molecules & MM/GBSA Kcal/mol & Ligand Efficiency \\
\hline Lansoprazole & -70.17 & -2.80 \\
\hline Omeprazole & -61.54 & -2.46 \\
Rabeprazole & -71.48 & -2.85 \\
Pantoprazole & -49.61 & -1.90 \\
\hline
\end{tabular}

Graph (4) shows MM/GBSA binding energies of different PPIs. It further shows that both Rabeprazole and Lansoprazole have highest stability throughout simulation time. Omeprazole showed a slight variation between $40 \mathrm{~ns}$ and $50 \mathrm{~ns}$ but becomes stable afterwards. Pantoprazole appears to be less stable among all PPIs throughout simulation time which has also been confirmed by the RMSD graph. 


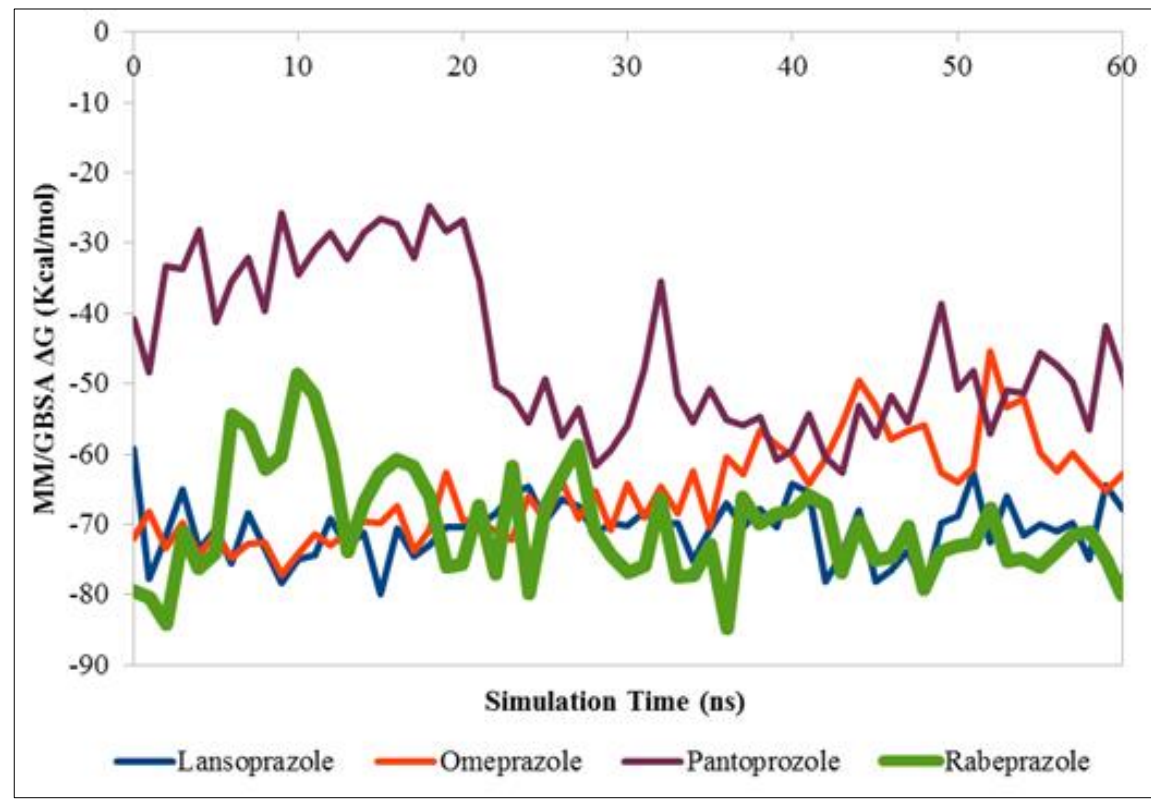

Graph 4. MM/GBSA binding energies of PPIs with dopamine D2 receptor.

$\mathrm{X}$-axis represents the simulation time (ns) while $\mathrm{y}$-axis shows the binding energies in $\mathrm{Kcal} / \mathrm{mol}$

\section{Conclusions}

In current study, it were screened four proton pump inhibitors for its interaction against human DRD2 receptor. Docking studies reveal that all PPI compounds have considerable affinity for human DRD2 receptor. Rabeprazole and Lansoprazole appears to have more binding affinities as compared to the other two. Additionally, MD simulations studies also indicate that the binding complex systems between PPIs and DRD2 receptors are stable enough. Hence, we can conclude that the PPIs have got the potential to interact with human DRD2 receptor and patients/individuals taking PPIs may show up an increase in serum prolactin levels leading to hyperprolactinemia and associated effects.

Acknowledgments: We are thankful to Schrodinger Team for granting us the license of Schrodinger Suite, enabling us to complete this study.

\section{References}

1.MISSALE C, NASH SR, ROBINSON SW, JABER M, CARON MG. Dopamine Receptors: From Structure to Function. Physiological Reviews., 78(1), 1998, 189-225.

doi:10.1152/physrev.1998.78.1.189

2.BHATIA A, LENCHNER JR, SAADABADI A. Biochemistry, Dopamine Receptors., [Updated 2020 Apr 23]. In: StatPearls [Internet]. Treasure Island (FL): StatPearls Publishing; 2020 Jan-. Available from: https://www.ncbi.nlm.nih.gov/books/NBK538242/

3.PIVONELLO R, FERONE D, LOMBARDI G, COLAO A, LAMBERTS SWJ, HOFLAND LJ. Novel insights in dopamine receptor physiology. European Journal of Endocrinology., 156(suppl_1), 2007, S13-S21. doi:10.1530/eje.1.02353

4.HABIBI M. Dopamine Receptors. Encyclopedia of Movement Disorders., 2010, 326-329. doi:10.1016/b978-0-12-374105-9.00236-7

5.GREGERSON KA. Mechanisms of Dopamine Action on the Lactotroph. In: Horseman N.D. (eds) Prolactin. Endocrine Updates, Springer, Boston, MA, 12, 2001.

6.HANSEN KA. Hyperprolactinemia and the Dopamine Receptor. US Endocrinology., 00(01), 2006, 74. doi:10.17925/use.2006.00.1.74 
7.DIAZ-TORGA G, FEIERSTEIN C, LIBERTUN C, GELMAN D, KELLY MA, LOW MJ, RUBINSTEIN M, BECUVILLALOBOS D. Disruption of the D2 dopamine receptor alters GH and IGFI secretion and causes dwarfism in male mice. Endocrinology,143, 2002, 1270-1279.

8.CRISTINA, C, GARCÍA-TORNADÚ I, DÍAZ-TORGA G, RUBINSTEIN M, LOW MJ, BECÚVILLALOBOS D. Dopaminergic D2 Receptor Knockout Mouse: An Animal Model of Prolactinoma. Pituitary Today: Molecular, Physiological and Clinical Aspects, 2006, 50-63. doi:10.1159/000094308 9.SHIN JM, SACHS G. Pharmacology of proton pump inhibitors. Curr Gastroenterol Rep., 10(6), 2008, 528-34.

10.STRAND DS, KIM D, PEURA DA. 25 years of proton pump inhibitors: A comprehensive review. Gut Liver., 11(1), 2017, 27-37.

11.SHIN JM, CHO YM, SACHS G. Chemistry of covalent inhibition of the gastric $(\mathrm{H}+, \mathrm{K}+)$-ATPase by proton pump inhibitors. J Am Chem Soc., 126(25), 2004, 7800-11

12.CHENG, FU-CHOU \& HO, YUNN-FANG \& HUNG, LO CHIAO \& CHEN, C \& TSAI, TUNGHU. Determination and pharmacokinetic profile of omeprazole in rat blood, brain and bile by microdialysis and high-performance liquid chromatography. Journal of chromatography. A. 949, 2002, 35-42. 10.1016/S0021-9673(01)01225-0.

13.ORTIZ-GUERRERO G, AMADOR-MUÑOZ D, CALDERÓN-OSPINA CA, LÓPEZ-FUENTES D, NAVA MESA MO. Proton Pump Inhibitors and Dementia: Physiopathological Mechanisms and Clinical Consequences. Neural plasticity, 2018, 5257285. https://doi.org/10.1155/2018/5257285

14.ROJO LE, ALZATE-MORALES J, SAAVEDRA IN, DAVIES P, MACCIONI RB, Selective interaction of lansoprazole and astemizole with tau polymers: potential new clinical use in diagnosis of Alzheimer's disease, J Alzheimers Dis. 19(2), 2010, 573-589. https://doi.org/10.3233/JAD-2010-1262

15.DANIELS IR, LAYER GT. How should gynaecomastia be managed? ANZ Journal of Surgery, 73(4), 2003, v, 213-216. https://doi.org/10.1046/j.1445-1433.2002.02584.x

16.MOHANTY BK, CHOUDHURY AK, BALIARSINHA AK. Study of Clinical Presentations of Patients with Hyperprolactinaemia Visiting a Tertiary Care Hospital. J Evid Based Med Healthc, 3(53), 2016, 2745-7.

17.DEEPINDER F, BRAUNSTEIN GD. Drug-induced gynecomastia: An evidence-based review. Expert Opin Drug Saf., 11(5), 2012, 779-95.

18.LA TORRE D, FALORNI A. Pharmacological causes of hyperprolactinemia. Ther Clin Risk Manag., 3(5), 2007, 929-51.

19.ARAKAWA R, OKUMURA M, ITO H, TAKANO A, TAKAHASHI H, TAKANO H, Positron emission tomography measurement of dopamine D2 receptor occupancy in the pituitary and cerebral cortex: Relation to antipsychotic-induced hyperprolactinemia. J Clin Psychiatry, 71(9), 2010, 1131-7.

20.FITZGERALD P, DINAN TG. Prolactin and dopamine: What is the connection? A Review Article. J Psychopharmacol., 22(2 SUPPL.), 2008, 12-9.

21.WANG S, CHE T, LEVIT A, SHOICHET BK, WACKER D, ROTH BL. Structure of the D2 dopamine receptor bound to the atypical antipsychotic drug risperidone. Nature., 555(7695), 2018, 26973.

22.MADHAVI SASTRY G, ADZHIGIREY M, DAY T, ANNABHIMOJU R, SHERMAN W. Protein and ligand preparation: Parameters, protocols, and influence on virtual screening enrichments. J Comput Aided Mol Des., 27(3), 2013, 221-34.

23.BAS DC, ROGERS DM, JENSEN JH. Very fast prediction and rationalization of pKa values for protein-ligand complexes. Proteins Struct Funct Genet., 73(3), 2008, 765-83.

24.JORGENSEN WL, TIRADO-RIVES J. The OPLS Potential Functions for Proteins. Energy Minimizations for Crystals of Cyclic Peptides and Crambin. J Am Chem Soc., 110(6), 1988, 1657-66. 25.***National Center for Biotechnology Information. PubChem Database. Lansoprazole, CID=3883, https://pubchem.ncbi.nlm.nih.gov/compound/Lansoprazole (accessed on Sep. 24, 2019).

26.***National Center for Biotechnology Information. PubChem Database. Rabeprazole, CID=5029, https://pubchem.ncbi.nlm.nih.gov/compound/Rabeprazole (accessed on Sep. 24, 2019). 
27.SHELLEY JC, CHOLLETI A, FRYE LL, GREENWOOD JR, TIMLIN MR, UCHIMAYA M. Epik: A software program for $\mathrm{pKa}$ prediction and protonation state generation for drug-like molecules. $\mathrm{J}$ Comput Aided Mol Des., 21(12), 2007, 681-91.

28.FRIESNER RA, BANKS JL, MURPHY RB, HALGREN TA, KLICIC JJ, MAINZ DT. Glide: A New Approach for Rapid, Accurate Docking and Scoring. 1. Method and Assessment of Docking Accuracy. J Med Chem., 47(7), 2004, 1739-49.

29.FRIESNER RA, MURPHY RB, REPASKY MP, FRYE LL, GREENWOOD JR, HALGREN TA. Extra precision glide: Docking and scoring incorporating a model of hydrophobic enclosure for proteinligand complexes. J Med Chem., 49(21), 2006, 6177-96.

30.SHAW D. Desmond Molecular Dynamics System, v 3.0. Schrödinger, New York, NY. 2011;

31.HOOVER WG. Canonical dynamics: Equilibrium phase-space distributions. Phys Rev A., 31(3), 1985, 1695-7.

32.MARTYNA GJ. Remarks on Constant-temperature molecular dynamics with momentum conservation. Phys Rev E., 50(4), 1994, 3234-6.

Manuscript received: 15.08 .2020 